\title{
Molecular cloning and sequence analysis of yps-3, a yeast-phase-specific gene in the dimorphic fungal pathogen Histoplasma capsulatum
}

\author{
Elizabeth J. Keath and Fatima E. Abidi
}

Author for correspondence: Elizabeth J. Keath. Tel: +1314 658 3965. Fax: +1 3146583117.

Department of Biology, Saint Louis University, St Louis, MO 63103, USA

\begin{abstract}
Genes specifically expressed in the parasitic yeast phase of Histoplasma capsulatum have been cloned to clarify the mechanisms underlying both pathogenesis and morphogenesis in this human dimorphic fungal pathogen. Previous studies have determined that the yeast-phase-specific gene, yps-3, is expressed differentially in two non-isogenic strains which differ in their thermotolerance and virulence. We have cloned the yps-3 homologues from the high virulence (G217B) and low virulence (Downs) strains, and obtained a partial CDNA clone representing the expressed gene from $H$. capsulatum G217B. The Downs clone harbours a 287 bp insertion sequence that disrupts a long ORF defined by the yps-3 G217B CDNA. Although the insertion sequence contains features reminiscent of mobile genetic elements, including 15 bp direct repeats of flanking sequence, it is not randomly distributed in the $H$. capsulatum genome. $S 1$ nuclease analysis was utilized to map the 5 ' end of the expressed yps-3 gene in G217B to potential regulatory regions which are largely homologous in both strains. This finding may point to a deficiency in a temperature inducible regulatory protein in the low virulence, temperaturesensitive Downs strain. The nucleotide sequence of the yps-3 gene and the predicted amino acid sequence of its product represents the first report of phase-specific gene and protein sequences in this widely distributed fungal pathogen. Further analysis of the product encoded by the yps-3 gene may provide significant insight into the pathogenic repertoire of $H$. capsulatum.
\end{abstract}

Keywords: Histoplasma capsulatum, yps-3, yeast-phase-specific genes, cloning

\section{INTRODUCTION}

Yeast-phase-specific $(y p s)$ genes which are potentially related to virulence or morphogenesis, or both, have been isolated and cloned from Histoplasma capsulatum, a dimorphic human fungal pathogen (Keath et al., 1989a). The organism exists as a multicellular mycelium in rich soils and organic matter in temperate environments worldwide, and proliferates as a unicellular yeast in infected host tissues (Wheat, 1988; Maresca \& Kobayashi, 1989). Clinically inapparent or mild disease can result from limited, primary site infection of $H$. capsulatum in the lungs, but an often life-threatening disseminated form of histoplasmosis can occur in immunodeficient patients, coding sequence; HTOYPSB) and L16845 (yps-3 mRNA, 3' end; HTOYPSC). particularly the elderly and groups with acquired immunodeficiency syndrome (AIDS) (Graybill, 1988; Wheat et al., 1990).

Since the yeast phase of the organism is the parasitic form, one approach to understanding virulence and/or morphogenesis has been to clone genes expressed specifically in the yeast phase at $37^{\circ} \mathrm{C}$. Expression of the previously cloned $y p s-3$ gene appears to correlate with virulence, temperature sensitivity, and the speed of the hyphal-toyeast transition in three non-isogenic $H$. capsulatum strains (Medoff et al., 1986b; Keath et al., 1989a). For example, the $y p s-3$ gene is expressed exclusively in the yeast phase of virulent strains, such as G217B, but is not transcribed in either the mycelial or yeast phases of low virulence strains, such as Downs or several similar isolates recently obtained from patients with AIDS (Spitzer et al., 1990). In G217B or other class 2 strains defined by analysis of various 
restriction fragment length polymorphisms in mitochondrial (Vincent et al., 1986), ribosomal (Spitzer et al., 1989), or nuclear genes (Keath et al., 1989b, 1992), the $y p s-3$ gene is expressed $1 \mathrm{~d}$ following an in vitro transition when mycelia grown initially at $25^{\circ} \mathrm{C}$ are induced to form yeast by shifting the temperature of incubation to $37^{\circ} \mathrm{C}$ (Keath et al., 1989a).

Virulence in $H$. capsulatum, and its relation to morphogenesis is of particular interest. Although conversion to the yeast phase is most probably required for progressive infection (Medoff et al., 1986a), the transition to the yeast form is not required for survival of the organism at an elevated temperature of $41{ }^{\circ} \mathrm{C}$ (Medoff et al., 1987). Genes essential for early adaptation to elevated temperatures or for the conversion to, and maintenance of, the parasitic phase are likely to represent significant virulence determinants. Adaptation to the macrophage or tissue environment of the host and transformation to the yeast state may entail the activation of temperature-sensitive promoters or control elements which may, in turn, be strongly influenced by alteration in temperature.

To further understand the genetic and regulatory processes in $H$. capsulatum, the genomic $y p s-3$ gene from G217B and the non-expressed homologue from the Downs strain have been cloned and sequenced. The Downs strain contains a repetitive 287 bp sequence which interrupts a long ORF defined by a G217B yps-3 cDNA clone. Although this sequence is flanked by two $15 \mathrm{bp}$ direct repeats, it does not function as a mobile genetic element in two different classes of $H$. capsulatum as evaluated by field inversion gel electrophoresis of chromosomal sized DNAs.

\section{METHODS}

Organisms and culture conditions. Stock cultures of $H$. capsulatum G217B (ATCC 26032) and the temperature-sensitive Downs strain (ATCC 38904) were maintained in liquid medium containing $2 \%(\mathrm{w} / \mathrm{v})$ glucose and $1 \%(\mathrm{w} / \mathrm{v})$ yeast extract at $25^{\circ} \mathrm{C}$ or $37^{\circ} \mathrm{C}$ for hyphal and yeast phases, respectively. For temperature sensitivity experiments, mycelia were induced to form yeast cells at $34^{\circ} \mathrm{C}$ in liquid culture.

Isolation of mRNA for Northern blot analysis and CDNA library construction. Total cellular RNA was isolated from the Downs and G217B strains of $H$. capsulatum by mechanical. disruption in UNSET buffer ( $8 \mathrm{M}$ urea, $2 \%$, w/v, SDS, $150 \mathrm{mM}$. $\mathrm{NaCl}, 100 \mathrm{mM}$ Tris/HCl, $\mathrm{pH} 7 \cdot 5$, and $1 \mathrm{mM}$ EDTA) by Braun homogenization. Nucleic acids were repeatedly extracted in phenol-chloroform-isoamyl alcohol (PCI), followed by ethanol precipitation. Following recovery, the RNA was resuspended in NSET buffer (UNSET buffer lacking urea), and digested with proteinase $\mathrm{K}\left(35 \mu \mathrm{g} \mathrm{ml}^{-1}\right.$, Boehringer Mannheim) for $30 \mathrm{~min}$ at $37^{\circ} \mathrm{C}$. The RNA was re-extracted with PCI and precipitated in ethanol. PolyA ${ }^{+}$RNA was obtained from both strains and phases by oligodT cellulose chromatography (Ono et al., 1980), electrophoresed with commercial RNA markers (Gibco BRL) in $1.1 \%(\mathrm{v} / \mathrm{v})$ formaldehyde agarose (Rave et al., 1979) and transferred to Nytran (Schleicher and Schuell) as previously described (Keath et al., 1992). The blot was hybridized under aqueous conditions ( $7 \% \mathrm{SDS}, 1 \% \mathrm{BSA}, 1 \mathrm{mM}$ EDTA, $0.5 \mathrm{M}$ sodium phosphate buffer) with $10^{6}$ c.p.m. of probe per $\mathrm{ml}$ of hybridization solution (Church \& Gilbert, 1984). Probes were prepared by nick-translation (Rigby et al., 1977) and included the previously described $1.85 \mathrm{~kb}$ HindIII fragment representing the G217B yps-3 gene (Keath et al., 1989a), and the $1.4 \mathrm{~kb}$ BamHI-HindIII fragment containing the cloned $H$. capsulatum actin gene (Spitzer et al., 1990). For cDNA library construction, SDS was omitted from the isolation procedure during the initial breakage and in the subsequent steps, since SDS appeared to inhibit the extent of first strand synthesis (unpublished observations).

Generation of a yeast-phase-specific subtraction library from G217B. A subtraction library enriched for G217B yeast-phasespecific sequences was prepared in the ampicillin resistant phagemid, pTZ (Invitrogen). Individual double-stranded cDNA libraries from the yeast and mycelial phases of G217B were generated by a modified Gubler-Hoffmann protocol (Gubler \& Hoffman, 1983) from $50 \mu \mathrm{g}$ polyA ${ }^{+} \mathrm{RNA}$, and inserted into the phagemid vector in the non-palindromic $B s t \mathrm{XI}$ site. Following transformation, single-stranded (ss) phagemid DNA from each library was rescued by infection with R408 helper phage. Photo-biotinylated ss phagemid DNA from the mycelial phase was hybridized with ss yeast phagemid DNA; hybrid cDNAs common to both phases were removed with streptavidin, followed by PCI extraction. The organic phase was back-extracted three times with TE buffer $(50 \mathrm{mM}$ Tris $/ \mathrm{HCl}$, $\mathrm{pH} 7 \cdot 5,1 \mathrm{mM}$ EDTA) and the aqueous phases were pooled prior to ethanol precipitation. The $y p s$ subtraction library was recovered following reverse transcription and transformation in Escherichia coli $\mathrm{DH} 5 \alpha$. Recombinants harbouring the $y p s-3$ gene were identified by colony filter hybridization (Maniatis et al., 1982), and the insert sizes of five clones were determined by gel electrophoresis of $X b a I$-digested mini-prep DNAs.

Dideoxy DNA sequencing of the genomic yps-3 homologues from the G217B and Downs strains of $\boldsymbol{H}$. capsulatum. The Downs and G217B $y p^{s-3}$ genes were sequenced by the dideoxy chain termination method (Sanger et al., 1977) using Sequenase version 2.0 (USB). Clones were sequenced in both directions by a combined approach using ligation of appropriate restriction fragments into M13mp18 and M13mp19 vectors, and by the formation of a series of unidirectional nuclease $\mathrm{Ba} / 31$ deletions (Maniatis et al., 1982) from both the Downs and G217B clones. The pTZ yps3.3 cDNA from strain G217B was sequenced in both directions using the $B a l 31$ deletion strategy.

S1 nuclease analysis of the $\mathbf{5}^{\prime}$ end of the yps-3 gene in G217B. A $1.35 \mathrm{~kb}$ HindIII-SmaI fragment from the genomic $y p s-3$ sequence was subcloned in both orientations in M13mp18 and M13mp19 vectors. Preliminary studies (data not shown) demonstrated that only the M13mp19 construct hybridized to $y p s-3$ transcripts in Northern blot analysis, and this construct was used in the protection experiments. The $1.35 \mathrm{~kb}$ HindIII-S mal probe was prepared by primer extension using $\left[{ }^{32} \mathrm{P}\right] \mathrm{dATP}$ and the Klenow fragment of DNA polymerase I (New England Biolabs). The probe was purified by separation on a urea polyacrylamide gel, and the radiolabelled fragment removed by electroelution in $1 \times \mathrm{TBE}$ buffer. The probe was hybridized (25000 c.p.m. per reaction) at $55^{\circ} \mathrm{C}$ overnight in $20 \mathrm{mM}$ Tris/ $\mathrm{HCl}, \mathrm{pH} 7 \cdot 4,0.4 \mathrm{M} \mathrm{NaCl}, 100 \mathrm{mM}$ EDTA and $10 \mathrm{mM}$ dithiothreitol to $2 \mu \mathrm{g}$ poly $\mathrm{A}^{+} \mathrm{RNA}$ from the yeast and mycelial phases of Downs and G217B. S1 nuclease (Sigma) digestion was performed at $37^{\circ} \mathrm{C}$ for $1 \mathrm{~h}$ in $1 \times \mathrm{S} 1$ buffer (40 units $\mathrm{S} 1$ nuclease, $0.3 \mathrm{M} \mathrm{NaCl}, 30 \mathrm{mM}$ sodium acetate, $\mathrm{pH} 4.6,1 \mathrm{mM} \mathrm{ZnSO}$, $20 \mu \mathrm{g}$ salmon sperm DNA ml${ }^{-1}$ ). Samples were electrophoresed on $6 \%$ urea polyacrylamide gels followed by autoradiography. Labelled pBR322 markers prepared by digestion with EcoRI and HinfI (Maniatis et al., 1982) were electrophoresed in parallel to determine the size of the protected fragment(s).

Field inversion gel electrophoresis (FIGE) of chromosomal sized DNA molecules from $\boldsymbol{H}$. capsulatum. Chromosomal sized 
DNAs from the $217 \mathrm{~B}$ and Downs strains of $H$. capsulatum were fractionated on $0.4 \%$ agarose gels by FIGE (Carle et al., 1986) using an embedded lysis technique modified for Histoplasma (Steele et al., 1989). Histoplasma DNAs were electrophoresed in parallel with chromosomal sized DNA obtained from Saccharomyces cerevisiae obtained by the method of Carle \& Olsen (1985). FIGE was performed under ramping conditions for $140 \mathrm{~h}$ at $35 \mathrm{~V}$. Initial conditions were established at $1200 \mathrm{~s}$ forward, $200 \mathrm{~s}$ reverse to a final setting of $3600 \mathrm{~s}$ forward, $1800 \mathrm{~s}$ reverse. The gel was treated with $0 \cdot 1 \mathrm{M} \mathrm{HCl}$, neutralized and then transferred to Nytran (Southern, 1975). The hybridization conditions were as described above.

\section{RESULTS AND DISCUSSION}

\section{Expression of the yps-3 gene in the virulent G217B and the low virulence Downs strain of $\boldsymbol{H}$. capsulatum}

Previous studies (Keath et al., 1989a) had suggested that expression of the $y p s-3$ gene correlated with thermotolerance and virulence in the yeast phases of three nonisogenic $H$. capsulatum strains cultured at $37^{\circ} \mathrm{C}$. It was possible, however, that the absence of $y p s-3$ gene expression in the Downs strain was due to temperature stress in this thermosensitive strain. As a result, gene expression was evaluated in the mycelial phase and in the yeast phase achieved following a temperature-induced transition at $34^{\circ} \mathrm{C}$. As seen in Fig. 1(b), yeast phase organisms of the $\mathrm{G} 217 \mathrm{~B}$ strain cultured at $34^{\circ} \mathrm{C}$ express

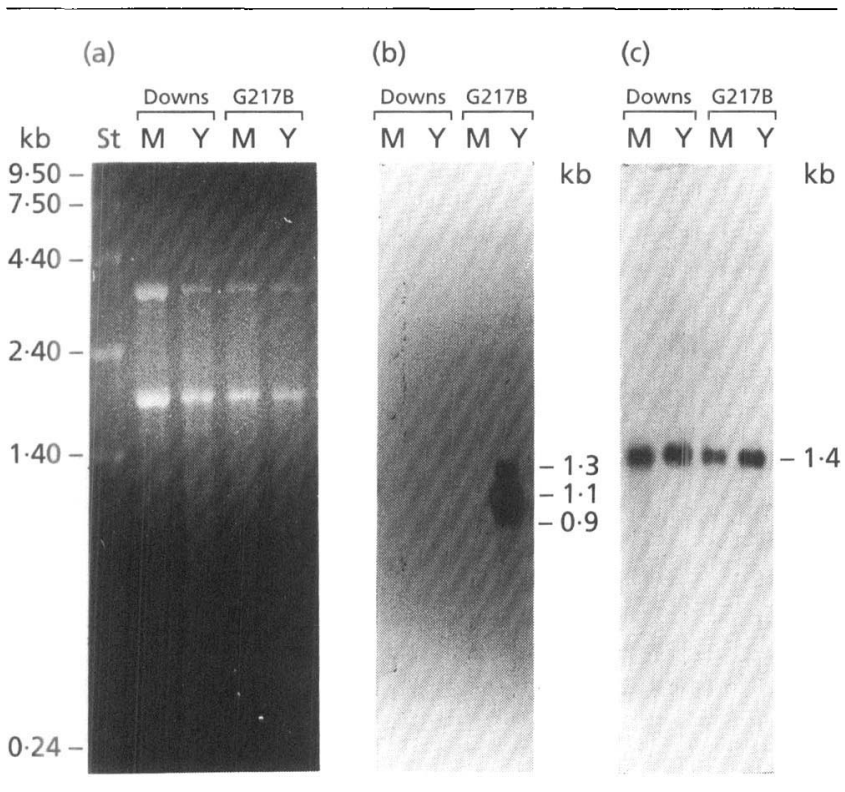

Fig. 1. Expression of the yps-3 gene in the Downs and G217B strains of $H$. capsulatum at $34^{\circ} \mathrm{C}$. PolyA ${ }^{+}$-selected RNAs from the Downs (low virulence) and G217B (high virulence) strains were electrophoresed in $1.1 \%$ formaldehyde agarose containing ethidium bromide $\left(1 \mu \mathrm{g} \mathrm{ml}^{-1}\right)$ with a commercial RNA ladder (Gibco BRL, St). The ethidium bromide stained gel (a), was transferred and hybridized in (b) with the $1.85 \mathrm{~kb}$ HindlII genomic yps-3 fragment from G217B in an aqueous buffer at $65^{\circ} \mathrm{C}$. Filters were washed once in $2 \times \mathrm{SSC} / 0.1 \%$ SDS at room temperature and at $65^{\circ} \mathrm{C}$ in $0.1 \times \mathrm{SSC} / 0.1 \%$ SDS. Following exposure, the blot was stripped and re-hybridized in (c) with the $1.4 \mathrm{~kb}$ BamHI-HindIII fragment containing the cloned Histoplasma actin gene. $M$, mycelial phase; $Y$, yeast phase. The sizes of the molecular mass standards are given in $\mathrm{kb}$. (a)

(i)

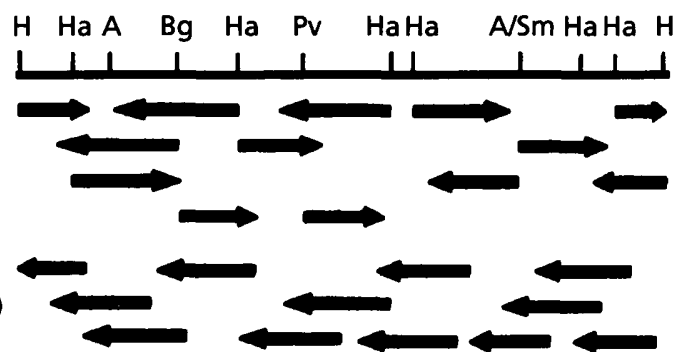

(i)

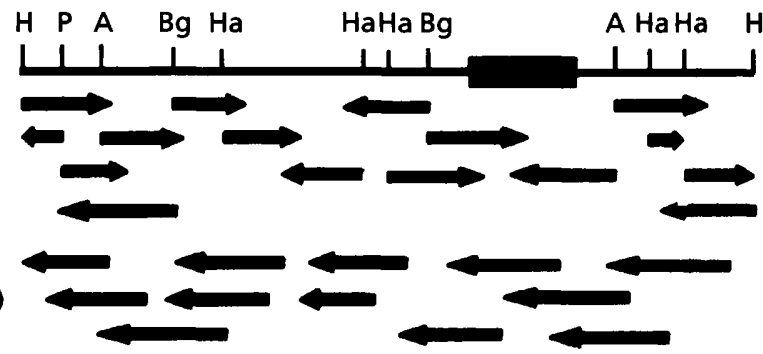

$100 \mathrm{bp}$

Fig. 2. Restriction analysis and DNA sequencing strategy for the genomic $1.85 \mathrm{~kb}$ HindIII G217B (a) and the $2.1 \mathrm{~kb}$ HindlII Downs (b) yps-3 gene homologues. The restriction maps of the G217B (a) and Downs (b) clones were determined by Southern analysis. The Downs sequence carries a repetitive element designated by the black box. The G217B yps-3 gene was sequenced by both the restriction fragment strategy $[a(i)]$ and by $B a / 31$ unidirectional deletions [a(ii)]. The genomic Downs sequence was determined as shown in [b(i)] and $[b(i i)]$ by the restriction fragment and deletion methods, respectively. The extent and direction of the determined sequences are indicated by arrows. A, Aval; Bg, Bg/ll; H, HindlII; Ha, Haelli; P, Pstl; Pv, Pvull; Sm Smal.

the $y$ ps- 3 gene as three transcripts ranging in size from 0.9 to $1.3 \mathrm{~kb}$. In contrast, the gene is not transcribed in either yeast (grown at $34^{\circ} \mathrm{C}$ ) or in mycelial forms of the Downs strain. The absence of phase-specific gene expression in Downs was not due to RNA degradation or transfer artifacts, since the staining in Fig. 1(a) is roughly equivalent for all tested RNAs, and since the $1.4 \mathrm{~kb}$ BamHI-HindIII fragment containing the Histoplasma actin gene probe hybridizes to transcripts of $1.4 \mathrm{~kb}$ in all lanes (Fig. 1c).

\section{DNA sequence analysis and comparison of the Downs and G217B genomic homologues of the yps-3 gene}

Although the $y p s-3$ gene was not expressed in the yeast phase of the low virulence Downs strain at either $34^{\circ} \mathrm{C}$ or $37^{\circ} \mathrm{C}$, previous studies have demonstrated that the gene was not deleted from the genome but was carried on a larger $2 \cdot 1 \mathrm{~kb}$ HindIII fragment (Keath et al., 1989a,b). The Downs $y p s-3$ clone was obtained by screening a genomic 


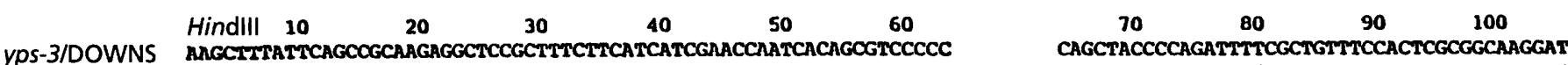
yps-3/DOWNS ANGCTTATTCAGCOGCAAGAGGCTCCACTTTCTTCATCATCGAACCAATCACAGCGCCGCCCCCCCCCCCCCAGCTACCCCAGATTTTGGCTACTTCCACTCGCGGCAAGGAT

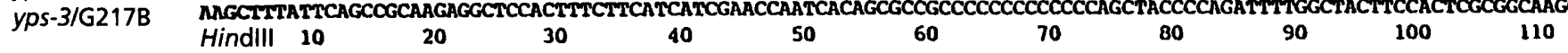

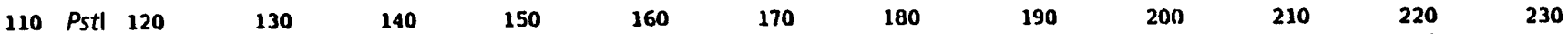
CGCCOGA CTGCACCCTCAACTCAACGACATCACCACCATOGTCAGGATGCATAGCCGAAATCTTGATAACAATGCACG AGGACAGTACNTGGCTATTGCTTTGCTGGTTCGTCCCATCGACTCTGAT CGCCOGATTGCATCCCCAACTCAACGACATCACCACCATCGTCAGGATGCATGGCCGAAGTTTTGATAACAATTCACGGAGGACAGTTCATGGCTATTGCTTTGCTGGTTCGTCCCATCGACTCTGAT

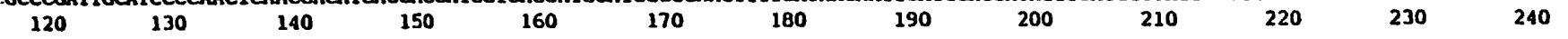

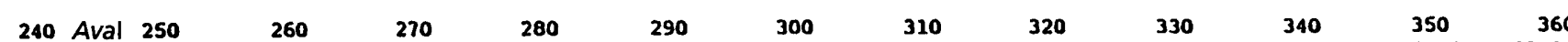

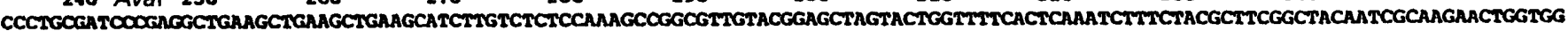
CCCTGCGA TCOOAGGCTGAAGCTGAAGCTGAACCATCTTGTCTCTCCAAAGCTGGCOTTGTACGGAGCTAGTCCTGGTTTCACTCAAATATTTCTACGGTTCGGCTACGATCGCAAGAACTGGTGG

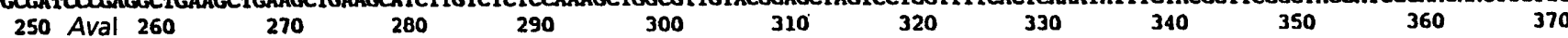

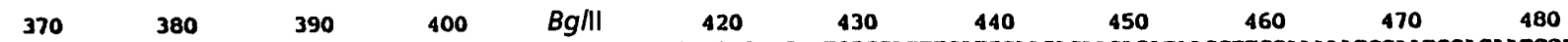
CTACTTCCAATGGAATACATACAGGTCGTACATTCCTCGATGTTCGGCNGATCTGGAAGTGTTGTATCTTGATTTCATTCANGACAACAGATAACCTTGGAAAATCGAATCGAGAATCCAGGACGAC CTACTTCCAATGGAATACATACAGGTCGTACATTCCTCGATGT CGGCNGATCTGGAAGTGTTGTATCTTGATTCCATTCAAG C ACAGATACCCTGGCANAATCGANTCGAGAATCCAGGATGAC

$\begin{array}{cccccccccccc}380 & 390 & 400 & 410 & B g / l l & 430 & 440 & 450 & 460 & 470 & 480 & 490 \\ 500 & 510 & 520 & 530 & 540 & 550 & 560 & 570 & 580 & 590 & 600 & \end{array}$

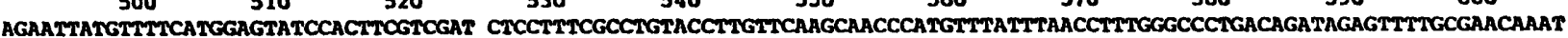
AGAATTATGTTTCATGGATTATCCACTTCGTCGATTCGCCTTTCCCCTGTACC

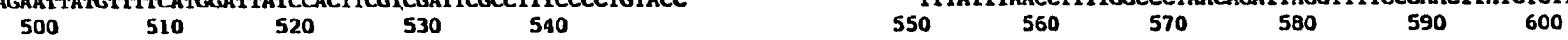

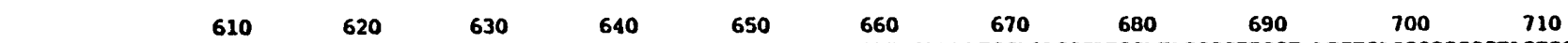

AATTCGTTCGCTTTGCGACATTTTCEGTGCTCTCTTGATCGTCCATTCAGCTCATTGANAATCCACAGCCATGCATAGCCCTTCGT ATGTGACGGGGCGCTAGTGGTCTAGG CATCCCTAATA CTTTAATTCGTCGCTTTACGACATCTTOGOTGCTCTCTTGATCGTCCATTCAGCTCATTGAAAATCCACAGCCATGCATAGCCCTTCGTAATGTGACCGGGAGCTAGTCGTCTAGG

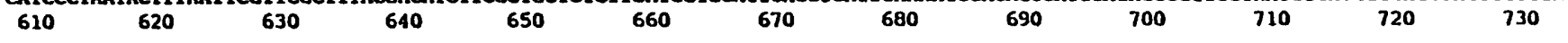

$\begin{array}{lllllllllllll}720 & 730 & 740 & 750 & 760 & 770 & 780 & 790 & 800 & 810 & 820 & 830 & 840\end{array}$

COTG TTCATAGATGGTTCGATAAGTCACCAAGTNGCAGCTTPACCACAGCGCAGTGATGCAATAATGTGTTACCACAGCTTCTTGCTACGAAGGCAACTCTGAGTACTGGTACACTGCCCATACTTC CGTGATTCATAGATGTTLOGATAGT CAAGTTCAGCTGTACCACAGCGCAGTGATGCANTAATGTGTTACCCCAGCTTCTTGCAACGATGCAACTCTAAGTACTGGTACACACAG CC $\begin{array}{lllllllll}740 & 750 & 760 & \text { PVull } & 780 & 790 & 800 & 810 & 820\end{array}$

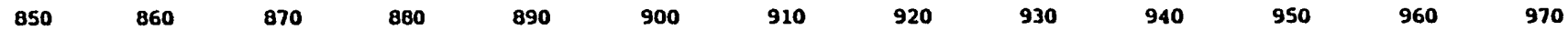
CCATTGATTTCCCTAGTGTGTCGACAGACGTCATATATTATTCTATTTTGAATTTACTCTTGTCCATGTTTCTGTTGCTGGCATCAGTTAGCCTCCCCATGATGAGACACTGCCTATTGAATGGAC CCATTAATTTCCCTAGTTTTTAGACAGACGTCATATATTATTCTATTTTGAATTTACTCTTGTCCATGTTTCTATTTCTAGCATCAGTTAGCCTGCCCATGATGAGACACTGCCTATTGAATGGAC

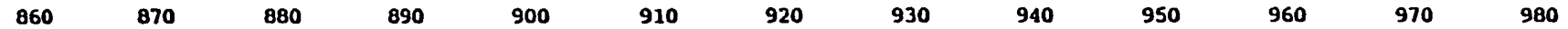

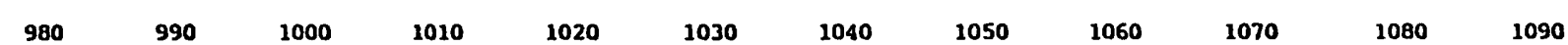
ACGTITTTGAACATCCCCTATCAGGAACCAGCCGGGTACGATGCTGGAGAGGCGTTGAAAGCAGGCCACGGAGAAGCCTCAAAGATATAAAAAGGTUC GAATTTGCTCTCTTTTTACTTCTTCTTTT AGGTITTRAACATCGCCTATCAGGAACCAGGCGGOTATGATGCTAAAGAGGGGTTAAAGCAGGCCATGGAGAAGGCTCGAAGATATAAAAAGGTGCGGA TTTCCTCTCTTTTYA TTCTTCTTTT

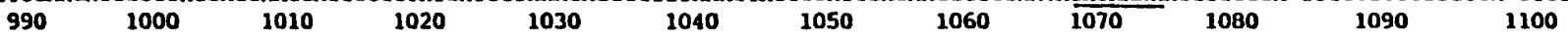

Fig. 3. For legend see facing page.

library generated from this strain (Harris et al., 1989) using the $1.85 \mathrm{~kb}$ G217B HindIII fragment as a probe. Detailed restriction endonuclease analysis was performed on both the G217B and Downs clones as described in Fig. 2(a) and (b), respectively. The HindIII fragments from the two strains differed with respect to several restriction endonuclease cleavage sites: the $1.85 \mathrm{~kb}$ G217B HindIII fragment harboured sites for PvuII and a single $B g / \mathrm{II}$ site, while the Downs $y p s-3$ homologue had two $B g / I I$ sites, but had recognition sites for PstI. The greatest restriction fragment length polymorphisms mapped to a HaeIIIAval fragment which was $295 \mathrm{bp}$ and approximately $780 \mathrm{bp}$ in the G217B and Downs homologues, respectively (Fig. 2a and b, data not shown). These preliminary findings suggested that the Downs clone might carry an insertion sequence which was absent in the G217B yps-3 gene.

Based on these observations, a sequencing strategy was developed for the HindIII fragments from both strains as detailed in Fig. 2. A series of overlapping deletions were generated for both clones by nuclease $\mathrm{Ba} / 31$ digestion followed by linker ligation and insertion into M13mp18 or mp19 series vectors [Fig. $2 \mathrm{a}$ (ii) and $\mathrm{b}(\mathrm{ii})$ ], additional sequence was confirmed by fragment subcloning in the same vectors [Fig. $2 a(i)$ and $b(i)$ ]

Comparative DNA sequence analysis of the Downs and G217B $y p s-3$ homologues (Fig. 3) indicated that the two genes were over $92 \%$ homologous in the first $1370 \mathrm{bp}$, mapping from the HindIII site (at position 1); the majority of differences with respect to endonuclease cleavage sites were due to point mutations which disrupted or created novel sites when the two clones were evaluated (Fig. 3).

A unique sequence was observed in the Downs $y p s-3$ homologue at position 1376 , where an internally repetitive 15 bp element disrupted the homology shared between the two sequences. A CCCACCGACAAATA(C/T) motif was reiterated 13 times within this region. Moreover, the unique Downs sequence was flanked at the right-hand end by a 14 bp direct repeat (Fig. 3) of a CCCACGGACAAGTA sequence preserved in G217B and by a CCCTATTGATAAG(T/A)A direct repeat of the G217B sequence at the left-hand end.

In this study, DNA sequence analysis was used to compare the Downs and G217B clones in this upstream, noncoding region (Fig. 3). The TATA element was preserved in both sequences at positions 1058 and 1067, respectively. 


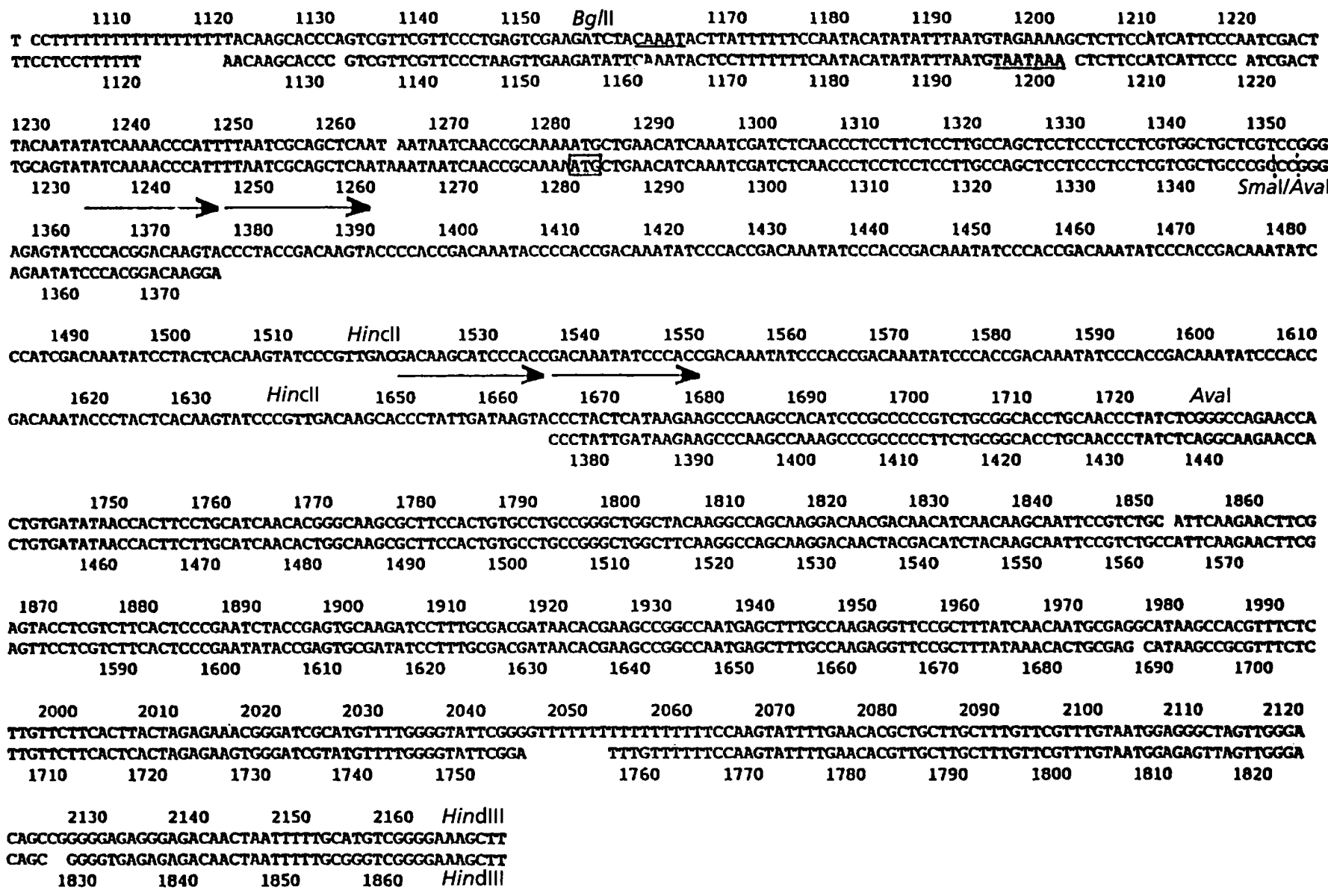

Fig. 3. Comparative DNA sequence analysis of the Downs (upper line) and G217B (lower line) yps-3 homologues. Both sequences are numbered from the first nucleotide of the upstream HindlII recognition sequence. There is extensive homology (greater than $92 \%$ ) in the initial 1370 bp of the sequences. A 287 repetitive element interrupts the Downs and G217B homology at position 1376 of the non-expressed sequence. The observed left-hand and right-hand direct repeats are indicated by the arrows. A 123 bp Hincll fragment was subsequently utilized as a repetitive-sequence-specific probe. A conserved TATA box (underlined) is found in both the Downs and G217B sequences at positions 1058 and 1067 , respectively.

The most significant difference in the Downs strain was an additional series of nine $T$ residues (positions 1102-1119, Fig. 3) which localized to the region immediately prior to the start site observed in G217B (see below). Several deletions ( $T$ ) and point mutations (CC in G217B replaced by TT in Downs) were also apparent in this region. These differences, although slight, may be sufficient for strainspecific $y p s-3$ gene expression. Alternatively, the Downs strain may be deficient in the synthesis of a temperature or stress inducible transcription factor which may recognize potential phase-specific promoters in both strains. Regulation of the expression or the levels of such transcription factors might play a significant role in the phase transition and in the morphogenic process. The discovery of additional yeast-phase-specific genes in the G217B strain which failed to be expressed in either phase of the Downs strain (unpublished observations) provides some preliminary support to this contention. However, the upstream regions of the Downs and G217B sequences may provide in vitro target binding sites for phase-specific transcription factors, and should be invaluable tools in the analysis of morphogenesis in $H$. capsulatum.

\section{The unique Downs element within the yps-3 sequence fails to function as a mobile element}

The unusual features of the Downs sequence with respect to redundancy and duplication of flanking sequence DNA suggested that the region might function as a mobile genetic element. An internal 123 bp HincII fragment (Fig. 3 ) contained within the novel sequence region of Downs was used as a probe for chromosomal sized DNA molecules separated by FIGE. Recent studies by Steele et al. (1989) have demonstrated the presence of three large chromosomal-like bands ranging from 2 to $7 \mathrm{Mb}$ in the G217B strain inferred from parallel electrophoresis of Schizosaccharomyces pombe and Saccharomyces cerevisiae. In contrast, the Downs strain harbours five chromosomes visualized by ethidium bromide staining and chromosome specific probes (Steele et al., 1989). In Fig. 4(a), the blot 
(a)

(b)
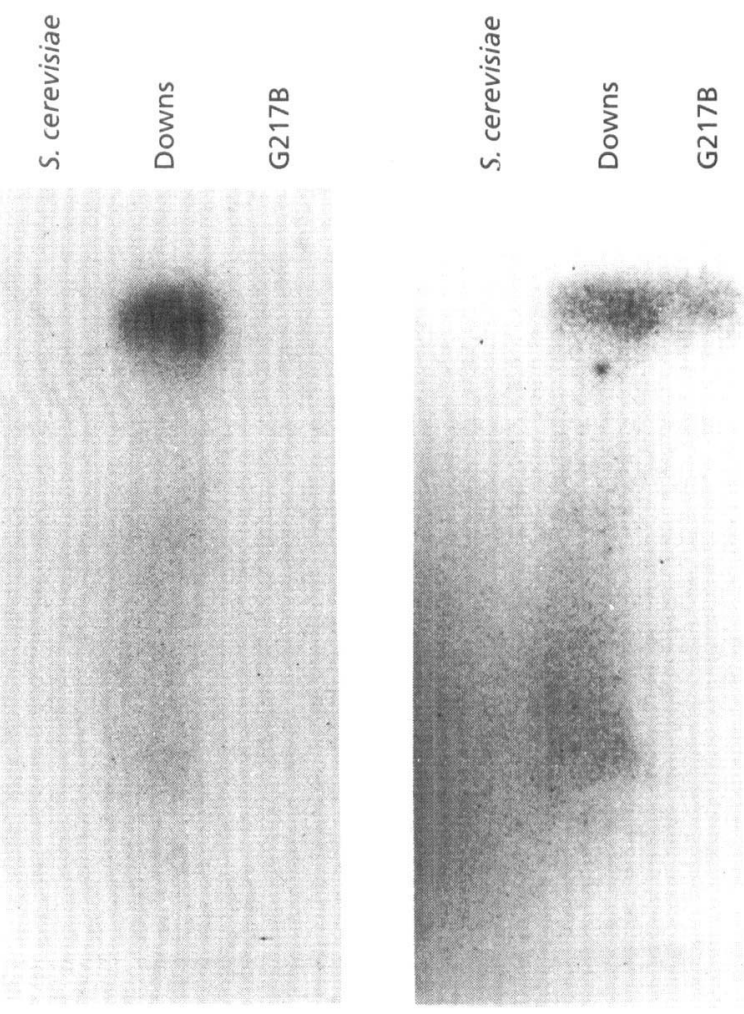

Fig. 4. Distribution of the reiterated sequence element on chromosomal sized molecules obtained from the Downs and G217B strains. The FIGE blot was hybridized in (a) under aqueous conditions to the $123 \mathrm{bp} \mathrm{Hincll}$ fragment specific for the repeated sequence identified by Downs sequence analysis. No hybridization was observed in the G217B or in the $S$. cerevisiae lanes; only one of five chromosomal sized molecules in the Downs strain harboured the element. In (b) the blot was stripped and rehybridized with the $1.85 \mathrm{~kb}$ HindIII G217B yps-3 sequence which lacked the repetitive sequence. The repetitive element and the yps-3 homologue co-migrate in the Downs strain.

containing both chromosomal sized DNA preparations from Downs and G217B was hybridized to the $123 \mathrm{bp}$ HincII fragment specific for the Downs insertion sequence; the probe reacted with a single chromosomal sized molecule in the Downs strain and failed to hybridize to any of the three chromosomal bands observed in G217B. Even under prolonged exposures, the probe failed to hybridize to any additional putative chromosomes in either strain, suggesting that the repetitive element was not randomly distributed among $H$. capsulatum chromosomes. To verify that the $123 \mathrm{bp} H$ incII fragment was associated with the Downs chromosome harbouring the $y p s-3$ homologue, the $1.85 \mathrm{~kb} H$ indIII fragment from $\mathrm{G} 217 \mathrm{~B}$ which lacked the repeated sequence was used to probe the stripped membrane filter (Fig. 4b). The $y p s-3$ gene probe lacking the novel sequence hybridized to single chromosomes in both the G217B and Downs strains and the reactive band in Downs comigrated with the chromosome identified with the repetitive element probe. These findings, as well as additional Southern blot experiments with EcoRI, BamHI, and $\mathrm{XbaI}$ (data not shown) suggested that the $287 \mathrm{bp}$ sequence found in the Downs strain was unique to this low virulence isolate, and that the element was not distributed randomly in the $H$. capsulatum genome.

The origin of this repetitive element in the Downs strain is unclear. The sequence does not function as a mobile genetic element, and the redundant nature of the region would have minimal coding capacity. One tempting derivation for such a repetitive sequence might be as a telomere (Blackburn, 1991). Healing of chromosomes by telomerase is known to play a role in the DNA sequence rearrangements described for developmentally regulated organisms such as Tetrabymena (Yu \& Blackburn, 1991). Native telomeric sequences have been cloned recently and described for Histoplasma (Woods \& Goldman, 1993) and contain reiterations of a GGGT'TA sequence dissimilar from the 15 bp motif observed in the Downs sequence.

\section{DNA sequence analysis of a partial yps-3 cDNA clone from the G217B strain}

It was of interest to determine if the unusual sequence uncovered by genomic sequence and chromosome blotting techniques in the Downs strain contributed to the absence of yeast-phase-specific gene expression observed in the temperature-sensitive strain. A cDNA subtraction library enriched for yeast-phase-specific sequences from G217B in the pTZ phagemid was screened using the $1.85 \mathrm{~kb}$ HindIII fragment. Subclones of the largest pTZ $y p s-3.3$ cDNA were generated in M13mp18/M13mp19 vectors at relevant restriction endonuclease sites, and by a bidirectional Bal31 deletion series. The $3^{\prime}$ end of the $y p s-3$ gene of G217B was defined by a consensus polyAAUAAA adenylation signal and a polyA tail which extended approximately 200 bases beyond the HindIII site (Fig. 5) of the genomic clone. The sequence of the $773 \mathrm{bp}$ pTZ yps-3 cDNA was aligned with the genomic sequence of the G217B clone (Fig. 5). The long ORF of this cDNA was in-frame with an upstream ATG beginning at position 1280 of the $\mathrm{G} 217 \mathrm{~B}$ genomic sequence and boxed in Fig. 5. A UGA stop codon would terminate the $y p s-3$ protein product at position 1832 in the genomic G217B sequence, prior to the HindIII site, predicting a $y p s-3$ polypeptide of approximately $17 \mathrm{kDa}$. The insertion element found in the Downs clone disrupted this ORF within a putative codon for aspartic acid (triangle in Fig. 5). Thus, the unique Downs element did not map to potential upstream promoter sequences for the $y p s-3$ gene and, if expressed, would have significantly disrupted the coding capacity of the $y p s-3$ mRNA.

\section{$5^{\prime}$ end analysis of the expressed yps-3 gene in G217B}

The larger sizes $(1.3,1.1$ and $0.9 \mathrm{~kb})$ of the authentic $y p s-3$ transcripts detected in Northern blots (Fig. 1b) suggested that the pTZ $y p s-3.3 \mathrm{cDNA}$ was a partial clone which lacked the $5^{\prime}$ end of the mRNA. To determine the precise end of the gene and thus to localize potential upstream promoter sequences in the G217B and Downs clones, S1 nuclease analysis was performed to map the $5^{\prime}$ 
AAGCTTMATTCAGCCGCAAGAGGCTCCACTTTCTTCATCATCGAACCAATCACAGCGCCGCCCCCCCCCC

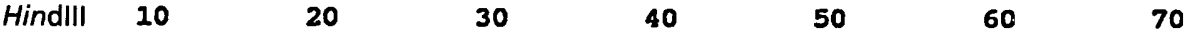

CCCAGCTACCCCAGATHTTGGCTACTTCCACTCGCGGCAAGGATCGCCCGATTGCATECCCAACTCAACG $\begin{array}{lllllll}80 & 90 & 100 & 110 & 120 & 130 & 140\end{array}$

ACATCACCA CCATCGTCAGGATGCATGGCCGAAGTTMTGATAACAATTCACGGAGGACAGTTCATGGCTA

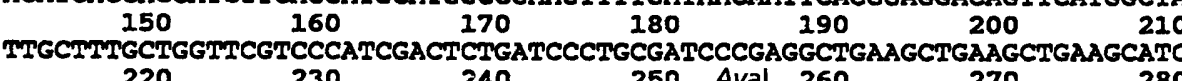

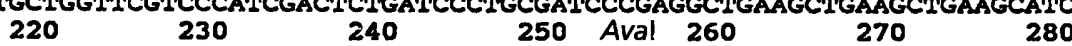

TTGTCTCTCCAAAGCTGGCGITGTACGGAGCTAGTCCTGGTTTCACTCAAATATTTCTACGCTTCGGCT

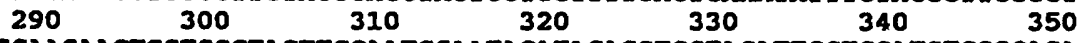

ACGATCGCAAGAACTGGTGGCTACTICCAATGGAATACATACAGGTCGTACATTCCTCGATGTCGGCAGA

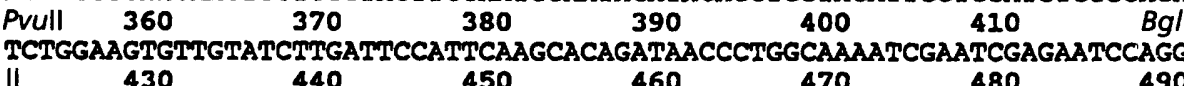

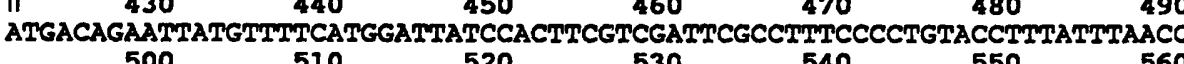

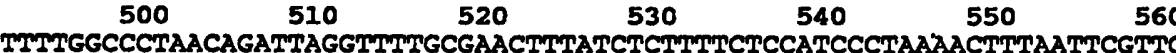

600

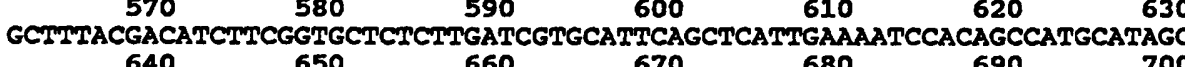

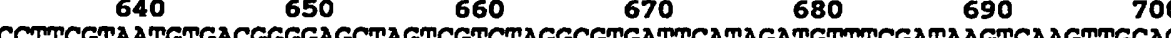

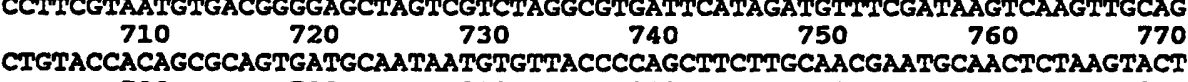

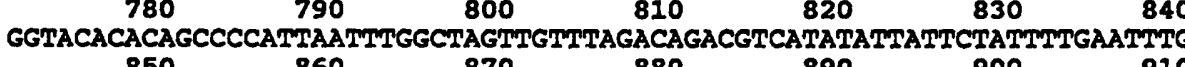

$\begin{array}{lllllll}850 & 860 & 870 & 880 & 890 & 900 & 910\end{array}$

ACTCTTGTCCATGTTTCTATITCTAGCATCAGTTAGCCTGCCCATGATGAGACACTGCCTATTGAATGGA

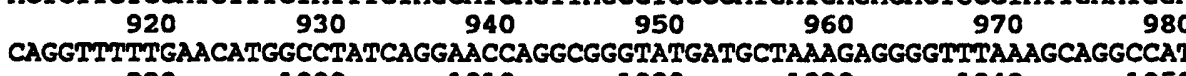

GGAGAAGGCTCGAAGATATAAAAAGGTGCGGATTTCCTCTCTTWTTATTCTTCTTTTTTCCTTCTTITT2

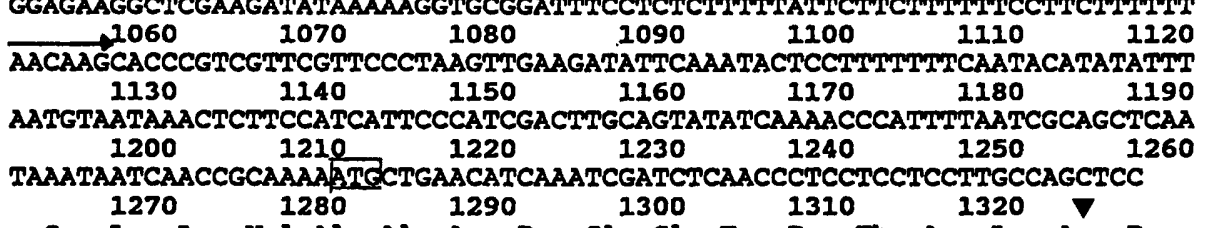

Ser leu Leu Val Ala Ala Arg Pro Gly Glu Tyr Pro Thr Asp Iys Asp Pro

TCC CTC CTC GTC GCT GCC CGC CCG GGA GAA TAT CCC ACG GAC AAG GAC CCT $1340 \quad$ Smal/Aval $1360 \quad 1370$

Ile Asp Lys lys Pro Lys Pro Lys Pro Ala Pro Phe Cys Gly Thr Cys Asn

ATT GAT AAG AAG CCC AAG CCA AAG CCC GCC CCC TIC TGC GGC ACC TGC AAC $13901400 \quad 1410 \quad 1420$

Pro Ile ser Gly Lys Asn His Cys Asp Ile Thr Thr ser Cys Ile Asn Thr

CCT ATC TCA GGC AAG AAC CAC TGT GAT ATA ACC ACT TCT TGC ATC AAC ACT $1440 \quad 1450 \quad 1460 \quad 1470 \quad 148$

Gly Lys Arg Phe His Cys Ala Cys Arg Ala Gly Phe Lys Ala Ser Lys Asp

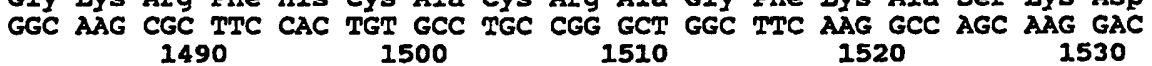

Asn Tyx Asp Ile Tyr Lys GIn Phe Arg Leu GIn Phe Lys Asn Phe Gly Phe AAC TAC GAC ATC TAC AAG CAA TTC CGT CTG CCA TTC AAG AAC TTC GAG TTC
1540

Leu val Phe Thr Pro Gly Tyr Thr Gly Cys Asp Ile Leu Cys Asp Asp Asn

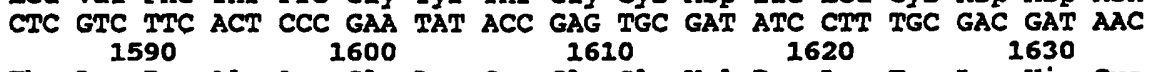

Thr Lys Pro Ala Asn GIy Leu Cys Gin Glu Val Pro Leu Tyr Lys His Cys ACG AAG CCG GCC AAT GAG CTI TGC CAA GAG GTT CCG CTT TAT AAA CAC TGC $\begin{array}{lccccc}1640 & 1650 & 1660 & 1670 & 1680\end{array}$

Gly His Lys Pro Arg Phe Ser Cys Ser Ser Leu Thr Arg Gly Val Gly Ser

GAG CAT AAG CCG CGT TTC TCT TGT TCT TCA CTC ACT AGA GAA GTG GGA TCG

$1690 \quad 1700 \quad 1710 \quad 1720 \quad 1730$

Tyr Val Leu Gly Tyr Ser Asp Leu Phe phe Pro Ser Ile Leu Asn Thr Leu

TAT GTT TTG GGG TAT TCG GAT TIG TTT TIT CCA ACT ATT TTG AAC ACG TTG

$1740 \quad 1750 \quad 1760 \quad 1770 \quad 1780$

Leu Ala Leu Phe Val Cys Asn Gly Glu Leu Val Gly Thr Ala Gly ***

CTT GCT TTG TTC GTT TGT AAT GGA GAG TTA GTT GGG ACA GCG GGG TGA

GAGAGACAACPAATMTTTECGGGTCGGGGAAGCTMCATCTATGTGCTCCAAATAGGGGTITTCGGGTTTC

$1840 \quad 1850 \quad 1860 \quad$ HindIII

CTATTGATAGCAATAAATACTGATGAAGTCGGACATAGGGAAACATCTTGTGTGCAAAATTATTTTAATAC

ATGGCTTAGACTCCTTTTGGAAAAA) 100

Fig. 5. Nucleotide and predicted amino acid sequence of the partial pTZ yps-3.3 CDNA aligned with the genomic G217B sequence. The long ORF of the 773 bp CDNA was oriented with respect to the genomic sequence, beginning at position 1328. The translated product was in-frame with the boxed ATG start codon at position 1280 of the genomic sequence. The predicted transcription start site inferred from $\mathrm{S} 1$ analysis is designated by an arrow; a TATAA element, and a putative ribosome binding site are found at positions 1067 and 1207, respectively. A consensus polyadenylation signal located downstream of the $3^{\prime}$ HindIII sequence is underlined and the putative stop codon is designated by asterisks. 


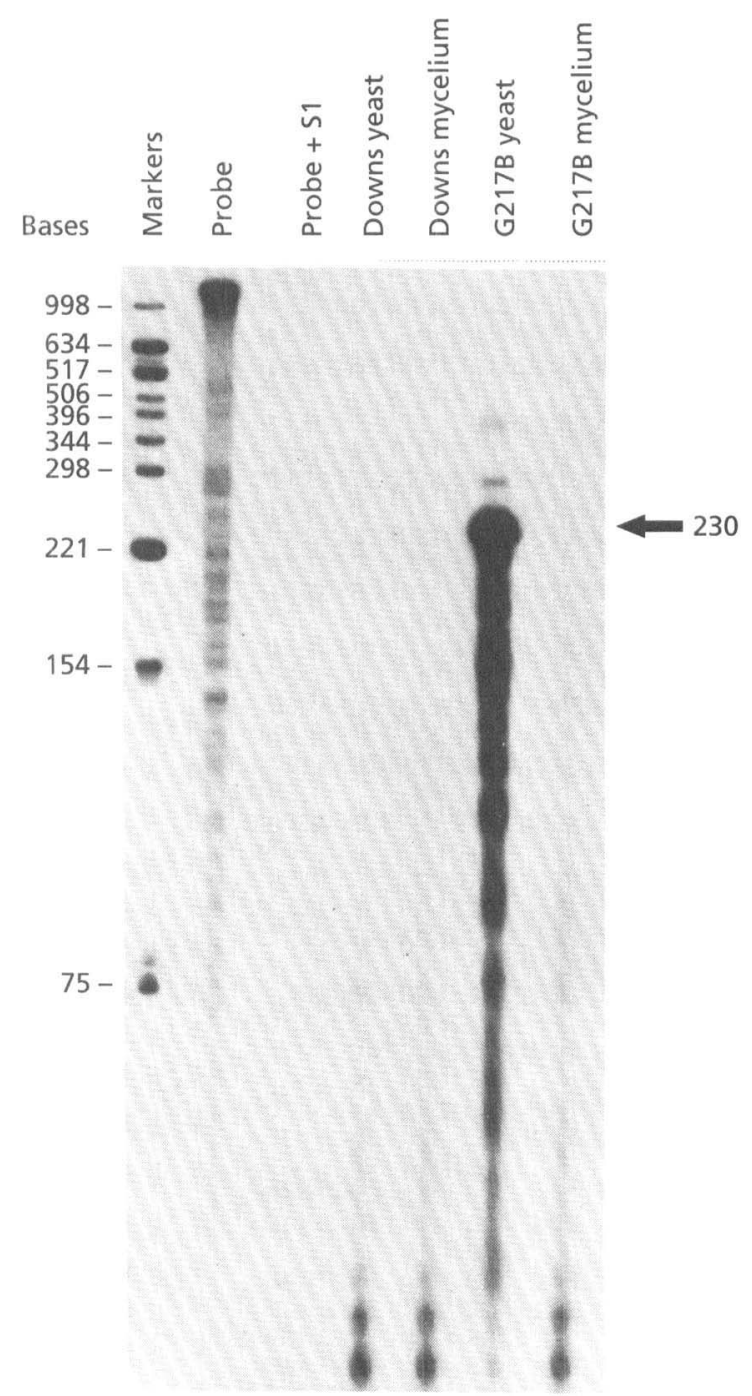

Fig. 6. S1 nuclease analysis of the $5^{\prime}$ end of the yps-3 gene. A $1.35 \mathrm{~kb}$ Hindll-Smal fragment was subcloned in M13mp19 for the nuclease analysis (see Fig. 2a). The single-stranded probe was hybridized to mycelial and yeast polyA ${ }^{+}$RNAs from the Downs and G217B strains. A major protected fragment of 230 nucleotides was obtained following S1 digestion in the G217B yeast sample. The full length probe fragment was observed in the control lane.

end of the $y p s-3$ gene. A $1.35 \mathrm{~kb}$ HindIII-SmaI fragment from the genomic $\mathrm{G} 217 \mathrm{~B}$ clone was uniformly labelled by primer extension within M13mp19 (Fig. 6a), processed by electroelution, and hybridized to mycelial and yeast polyA ${ }^{+}$RNAs from the Downs and G217B strains of $H$. capsulatum. The major start site of the gene mapped 230 nucleotides upstream of the $S \mathrm{maI}$ site exclusively in the G217B yeast tract (Fig. 6b), at position 1121 in the genomic G217B sequence. The putative purine start site was preceded in the genomic sequence by a region rich in $\mathrm{CT}$ bases, and was localized approximately 50 bases from a potential TATA box at positions 1067-1072 (Fig. 5). A consensus ribosome binding site (CCATCA) was located upstream of the putative ATG codon (Fig. 5) at position 1207. Neither the nucleotide sequences of the $y p s-3$ gene nor the predicted protein product of the cDNA are homologous to sequences currently in GenBank.

The yps-3 gene itself, expressed at high levels in the virulent G217B strain, may represent an important virulence determinant. Although the gene is not required for the transition from hyphal to yeast forms, it may play a role in facilitating recovery of the virulent strains from environmental or thermal stress. Further analysis of the $y p s-3$ gene product will be necessary to ascertain its role in virulence in G217B and other $H$. capsulatum strains, since only the yeast phase is known to survive within tissues or within macrophages (Eissenberg \& Goldman, 1991).

\section{ACKNOWLEDGEMENTS}

We are grateful to Dr Gerald Medoff, Dr George Kobayashi, and Dr Paul Steele for helpful discussions. The assistance of Mary Powderly and Susan Louw in the preparation of this manuscript is gratefully acknowledged. This research was supported by grant AI28950 from the US Public Health Service to E. J.K.

\section{REFERENCES}

Blackburn, E. H. (1991). Structure and function of telomeres. Nature 350, 569-573.

Carle, G. F. \& Olsen, M. V. (1985). An electrophoretic karyotype for yeast. Proc Natl Acad Sci US A 82, 3756-3760.

Carle, G. F., Frank, M. \& Olsen, M. V. (1986). Electrophoretic separation of large DNA molecules by periodic inversion of the electric field. Science 232, 65-68.

Church, G. M. \& Gilbert, W. (1984). Genomic sequencing. Proc Natl Acad Sci US A 31, 1991-1995.

Eissenberg, L. G. \& Goldman, W. E. (1991). Histoplasma variation and adaptive strategies for parasitism: new perspectives in histoplasmosis. Clin Microbiol Rev 4, 411-421.

Graybill, J. R. (1988). Histoplasmosis and AIDS. J Infect Dis 158, 623-626.

Gubler, U. \& Hoffman, B. J. (1983). A simple and very efficient method for generating cDNA libraries. Gene 25, 263-269.

Harris, G. S., Keath, E. J. \& Medoff, J. (1989). Expression of alpha and beta tubulin genes during dimorphic phase transitions of Histoplasma capsulatum. Mol Cell Biol 9, 2042-2049.

Keath, E. J., Painter, A. A., Kobayashi, G. S. \& Medoff, G. (1989a). Variable expression of a yeast-phase-specific gene in Histoplasma capsulatum strains differing in thermotolerance and virulence. Infect Immun 57, 1384-1390.

Keath, E. J., Painter, A. A., Travis, S. J., Kobayashi, G. S. \& Medoff, G. (1989b). DNA probe for the identification of Histoplasma capsulatum. J Clin Microbiol 27, 2369-2372.

Keath, E. J., Kobayashi, G. S. \& Medoff, G. (1992). Typing of Histoplasma capsulatum by restriction fragment length polymorphisms in a nuclear gene. J Clin Microbiol 30, 2104-2107.

Maniatis, T., Fritsch, E. F. \& Sambrook, J. (1982). Molecular Cloning: a Laboratory Manual. Cold Spring Harbor, NY: Cold Spring Harbor Laboratory.

Maresca, B. \& Kobayashi, G. S. (1989). Dimorphism in Histoplasma capsulatum: a model for the study of cell differentiation in pathogenic fungi. Microbiol Rev 53, 186-209.

Medoff, G., Maresca, B., Lambowitz, A., Kobayashi, G., Painter, A., Sacco, M. \& Carratu, L. (1986a). Correlation between patho- 
genicity and temperature sensitivity in different strains of Histoplasma capsulatum. J Clin Invest 78, 1638-1647.

Medoff, G., Sacco, M., Maresca, B., Schlessinger, D., Painter, A., Kobayashi, G. \& Carratu, L. (1986b). Irreversible block of the mycelial to yeast phase transition of Histoplasma capsulatum. Science 231, 476-479.

Medoff, G., Kobayashi, G. S., Painter, A. \& Travis, S. (1987). Morphogenesis and pathogenicity of Histoplasma capsulatum. Infect Immun 55, 1355-1358.

Ono, M., Cole, M. D., White, A. T. \& Huang, R. C. C. (1980). Sequence organization of cloned intracisternal A particle genes. Cell 21, 465-473.

Rave, N., Ckvenjakou, R. \& Boedtkar, H. (1979). Identification of procollagen mRNAs transferred to DBM paper from formaldehyde agarose gels. Nucleic Acids Res 6, 3559-3567.

Rigby, P. W. J., Dieckman, M., Rhodes, C. \& Berg, P. (1977). Labelling deoxyribonucleic acid to high specific activity in vitro with DNA polymeraseI. J Mol Biol 113, 237-251.

Sanger, F., Nicklen, S. \& Coulson, A. R. (1977). DNA sequencing with chain-terminating inhibitors. Proc Natl Acad Sci USA 74, 5463-5467.

Southern, E. (1975). Detection of specific sequences among DNA fragments separated by gel electrophoresis. J Mol Biol 98, 503-517.

Spitzer, E. D., Lasker, B. A., Travis, S. J., Kobayashi, G. S. \& Medoff, G. (1989). Use of mitochondrial and ribosomal DNA polymorphisms to classify clinical and soil isolates of Histoplasma capsulatum. Infect Immun 57, 1409-1412.
Spitzer, E. D., Keath, E. J., Travis, S. J., Painter, A. A., Kobayashi, G. S. \& Medoff, G. (1990). Temperature sensitive variants of Histoplasma capsulatum isolated from patients of acquired immunodeficiency syndrome. $J$ Infect Dis 162, 258-261.

Steele, P. E., Carle, G. F., Kobayashi, G. S. \& Medoff, G. (1989). Electrophoretic analysis of Histoplasma capsulatum chromosomal DNA. Mol Cell Biol 9, 983-987.

Wheat, L. J. (1988). Histoplasmosis. Infect Dis Clinics North Am 2, 841-859.

Wheat, L. J., Connolly-Stringfield, P. A., Baker, R. L., Curfman, M. F., Ends, M. E., Israel, K. S., Norris, S. A., Webb, D. H. \& Zeckel, M. L. (1990). Disseminated histoplasmosis in the acquired immunodeficiency syndrome: clinical findings, diagnosis, treatment, and review of the literature. Medicine 69, 361-374.

Woods, J. P. \& Goldman, W. E. (1993). Autonomous replication of foreign DNA in Histoplasma capsulatum: the role of native telomeric sequences. J Bacteriol 175, 636-641.

Vincent, R. D., Goewert, R., Goldman, W. E., Kobayashi, G. S., Lambowitz, A. M. \& Medoff, G. (1986). Classification of Histoplasma capsulatum isolates by restriction fragment length polymorphism. J Bacteriol 165, 813-815.

Yu, G.-L. \& Blackburn, E. H. (1991). Developmentally programmed healing of chromosomes by telomerase in Tetrabymena. Cell 67, 823-832.

Received 14 May 1993; revised 25 August 1993; accepted 20 October 1993. 